\title{
Psychosocial factors, cancer, and ischaemic heart disease
}

\section{H J Eysenck}

On 16 May we published an article by Pelosi and Appleby criticising the work of Eysenck and Grossarth-Maticek on psychological influences on cancer and ischaemic heart disease. Here Professor Eysenck responds
Institute of Psychiatry, University of London, London SE5 8AF H J Eysenck, professor emeritus of psychology

BMF 1992;305:457-9
Pelosi and Appleby recently criticised the extensive work of Ronald Grossarth-Maticek on psychosocial factors in the development of cancer and coronary heart disease, which began in the 'sixties and with which I have been associated during the past eight years. ${ }^{\prime}$ Constructive criticism is always welcome, but Pelosi and Appleby's paper had several faults which make it difficult to accept it as an objective account of what has been done, what is claimed, and what has been offered as evidence.

The important hypotheses investigated in the studies are listed in the box. Good evidence exists to support all these points, which find their theoretical basis in Sir William Osler's famous statement that it is often more important to know what person has the disease than what disease the person has. I have written a book presenting the evidence. ${ }^{6}$ Furthermore, there is independent evidence on most of these points, so that though Grossarth-Maticek's hypotheses were largely original when he started the studies in the 'sixties, they would now be considered commonplace. I will come back to this point later on.

\section{Poor criticisms}

Pelosi and Appleby suggest that description of methods, analyses, and results have been patchy and scattered widely "in obscure or unreferenced journals." Among these obscure or unreferenced journals are the Deutsche Zeitschrift für Onkologie, the Fournal of Psychosomatic Research, Social Science and Medicine, Cancer Detection and Prevention, and the American fournal of Epidemiology.

The critics consider that the questionnaire items devised by Grossarth-Maticek are "woolly" and "clumsy." Unfortunately, complex psychological theories cannot always be composed in simple language; Grossarth-Maticek thus decided to have the inventories applied by trained interviewers who could answer questions about meanings, assess relevant personal experiences of subjects, and help in the understanding of admittedly complex questions. As the inventories gave promising results as predictive instruments for the later occurrence of cancer and coronary heart disease, and have since received support from independent replications, they cannot have been as bad as suggested.

Other criticisms were so general as to defy attempts to see what they refer to. Thus they mention "many errors" and state that "essential details are missing from the results" and "the analyses are often inappropriate." Such general statements are meaningless and therefore cannot be refutated or discussed. The only example given is not encouraging for the critics. It refers to our first randomised trial of psychotherapy, where Pelosi and Appleby state that we "describe in detail how [we] individually matched 192 pairs of participants. But [we] report results on only 192 subjects...; either there have been no less than 10 elaborate misprints or misstatements in the description

\section{Hypotheses for Grossarth-Maticek's studies}

(1) Psychosocial factors (personality, stress) play an important part in the development of cancer and coronary heart disease

(2) These factors are different for cancer and heart disease and can be measured in healthy people, leading to the postulation of personalities prone to cancer or coronary heart disease

(3) The cancer prone personality is characterised by suppression of emotion and an inability to cope with interpersonal stress, leading to feelings of hopelessness, helplessness, and finally depression

(4) The coronary heart disease personality is characterised by strong reactions of anger, hostility, and aggression

(5) Appropriate behaviour therapy (stress management) can act prophylactically to make people prone to cancer or coronary heart disease less likely to develop either disease

(6) Similar types of treatment can prolong the life of patients with inoperable cancer

(7) Risk factors (psychosocial, smoking, genetic, etc) act synergistically, not additionally ${ }^{2}$

(8) Cancer and coronary heart disease are differentially influenced by coffee, cola, and alcohol, in line with theoretical expectation ${ }^{3-5}$

of the methods, or else there are no outcome data on exactly half the original participants." The answer of course is that no one was left out, we started with 192 subjects matched in pairs, and randomly assigned one of each pair to therapy and one to the control group. None of the referees considering the paper, nor the editor of the journal which published it, nor any of the numerous readers who corresponded with us had the slightest doubt about the design of the study.

They ask other questions to which the answers are so obvious that I wonder about the motivation behind them. Thus they ask "Why did the unfortunate control subjects in their randomised trials have such high mortality?" The reason, as stated in the paper, was of course that we chose subjects prone to cancer and coronary heart disease for the trial, for obvious reasons probands not prone to either disease on our questionnaires would have had too low a mortality in the period covered to give significant results. ${ }^{6}$

\section{Answered criticisms}

Another question is the reason for leaving the description of methods and analyses so vague; this implies that more details are needed but it is not stated what these details are. The question is therefore unanswerable. If the questions following this one are meant to illustrate the vagueness, they are easily answered. Thus the question "How many surveys were 
conducted in Heidelberg between 1972 and 1974," can be answered by stating that there were two such surveys in 1972, one of a largely random sample (except for age and sex constraints) and another of seriously stressed subjects. In 1973 there was another survey of several samples selected by different methods, the results from only some of which have been analysed. We hope to obtain sufficient funds to follow up and analyse all the subsamples studied in 1973.

Pelosi and Appleby ask: "Why did the type 1 and type 2 subjects have such low mortality in the investigation aimed at showing the dangers of psychoanalysis?" As stated in the article referred to by them, the term psychoanalysis now refers to a wide range of treatments, many of which would not have been considered proper psychoanalytic therapy by Freud. Patients were asked whether they considered that the treatment they received tended to make them more autonomous or less autonomous in their behaviour. This is relevant to Grossarth-Maticek's theory according to which healthy behaviour is associated with psychological autonomy. The study found that while treatment that decreased autonomy was correlated with higher rates of mortality, treatment that increased autonomy was not so correlated. The negative effects of psychoanalysis were stronger in those who had had two years or more of psychoanalysis than in those who had had less. Hence these are factors which reduce the negative effects of psychoanalysis overall, leaving a subgroup to show the result most strongly.

Pelosi and Appleby also ask how the personality typology was applied to participants in the early investigations.' From the beginning the healthy subjects constituting the sample were studied by $(a)$ a normative type questionnaire containing seven different traits, and $(b)$ a typological questionnaire specifying four types. Pelosi and Appleby state incorrectly that the four category typology was applied to the existing data later and that we could have made errors during reanalysis of data, reassigning individuals to personality types after the cause of death is known.

This is an unworthy suggestion. The two types of questionnaire (normative and quasi-ipsative) were developed at the same time of the basis of a clearly defined theoretical system, and their relation is clearly indicated in table VII of the orginal article. ${ }^{7}$ However, as we expected that the results would seem too good to be true to many sceptics we decided that the only decisive proof would be to continue the follow up for another four and a half years, to discover whether on the original questionnaires the correlation between type and mortality would be continued. The results were analysed by Dr H Vetter, who had all the original data; mortality assessment was supervised by Dr W-D Heller, then of the Karlsruhe Statistical Institute. The outcome showed conclusively that the same trends continued after the completion of the first 10 year

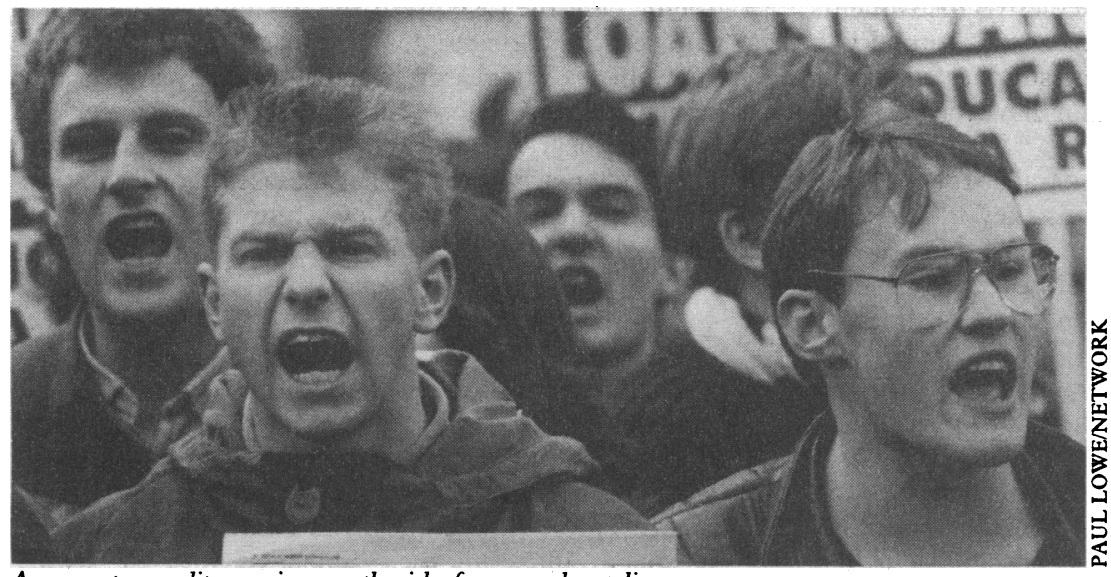

An angry personality may increase the risk of coronary heart disease follow up, thus showing the falsity of the PelosiAppleby hypothesis. ${ }^{8}$

\section{Rigorous analysis and investigation}

Vetter suggested a more refined test to exclude any possibility of prior knowledge having caused the observed correlation. ${ }^{9} \mathrm{He}$ put forward his "mortality hypothesis" - that prediction of deaths and cause of deaths from 1982 to 1986 might have been influenced by knowledge of existing relevant diseases in subjects. He tested this hypothesis by analysing deaths in which the disease was first discovered after the second follow up period had begun; even with a relatively small sample he found strong evidence that the prediction still held true. ${ }^{10}$ We now have evidence from the total sample studied, with the data collected independently by a Heidelberg clinic, that the data show a continued prediction accuracy well beyond any conventional levels of probability; these data are being written up and will be published. Clearly Pelosi and Appleby's suggestion is totally incorrect, and the data continue to show a high level of predictability.

Pelosi and Appleby complain that the numerous probands treated individually in groups by GrossarthMaticek suggest that "the amount of time spent by this single senior academic on his experimental psychotherapies is huge and certainly unprecedented.' Grossarth-Maticek devoted his whole life to this investigation, undertook no other duties, and worked 10 or more hours every day on this single topic for a long time. This cannot be compared with the small amount of time most busy consultants have to spare for research purposes.

It is a shame that Pelosi and Appleby asked their questions and made their accusations without contacting either Grossarth-Maticek or myself; most of them could have been clarified in direct discussion, and we could have directed them to important references they clearly did not consult. What is equally difficult to understand is their failure to mention the elaborate precautions taken by Grossarth-Maticek to guard against just the kind of criticisms produced by Pelosi and Appleby; some of these are listed in one of the papers they cited. " The precautions include depositing the names of probands in independent university departments, having the collection of mortality data checked by an independent observer, having a random sample of the 100 or more workers who collected the original and follow up data interviewed by an independent observer, depositing all the data in the computer of a highly critical statistician who carried out relevant analyses, and continuing follow up well beyond the original finishing date to rule out any contamination of data. Mention of these precautions is a necessary counter to the somewhat arbitrary accusations of Pelosi and Appleby.

\section{Too good to be true}

Let me next deal with the widespread notion that the results are "too good to be true." It is of course impossible to disprove such a statement because it is essentially unfalsifiable, and hence not a scientific statement at all. But what can be done is to show, as I tried to do elsewhere, ${ }^{12}$ that the Grossarth-Maticek correlations between personality and cancer are of the same order as earlier correlations; results reported by Kissen and Eysenck, ${ }^{13}$ and by Schmale and Iker ${ }^{14}$ are not out of line with those reported by GrossarthMaticek. I might also have expected our critics to note that independent authors using the Grossarth-Maticek instruments have found correlations between disease and personality, quite similar to those found by himfor example, the studies by Schmitz ${ }^{15}$ and Quander- 
Blaznik. ${ }^{16}$ And as regards the effects of psychotherapy, Spiegel $e t$ al have provided evidence of effects even better than those reported by Grossarth-Maticek. ${ }^{17}$ Omission of these important facts makes criticism on the grounds of results being "too good" look unfair and one sided.

This is not to deny that there are occasional faults in the methods and defects in data analysis, which may be inevitable in such a large undertaking. Thus in the original Yugoslav study the sample was made up of the oldest inhabitant in every second dwelling in a small Yugoslav town; several hundred highly stressed subjects were also included. This is clearly faulty methodology, and might suggest that the stressed group was added as an afterthought because the aged sample did not give the expected results. Spielberger reanalysed the original data for the groups separately, and found that results for the original sample were actually better when the stressed group was not included (personal communication). Vetter discovered in a reanalysis that a few questionnaires had identical endorsement patterns, suggesting that the student collecting this set of data had filled in the questionnaires without interviewing the probands. Separate analysis with these data excluded showed that this did not affect overall results. Occasionally people with identical surnames were confused in the analysis, but again when discovered it was found that this produced no change in the overall results. Careful and continued scrutiny of the data by Vetter, an experienced statistician, makes it unlikely that many such errors remain.

\section{Further research needed}

Pelosi and Appleby suggest that "there should be a total re-examination and proper analysis of the original data from this research." I fully agree. GrossarthMaticek has always been willing to allow competent critics access to his data; it is unnecessary for Pelosi and Appleby to ask again in terms that suggest unwillingness on the part of the investigator. Indeed both Grossarth-Maticek and I have always pressed for the replication of these studies, by an independent group, but with little success. One such study is now underway in Heidelberg, under the direction of Professor Amelang; I have already mentioned two smaller replication studies which have been published. The neglect of Grossarth-Maticek's work mentioned by Pelosi and Appleby' ${ }^{1}$ is a scandal in view of its obvious social importance. Their critical review, however incorrect, full of errors and misunderstandings, and lacking in objectivity, may have been useful in drawing attention to a large body of work, of both scientific and social relevance, that has been overlooked for too long.

1 Pelosi AJ, Appleby L. Psychological influences on cancer and ischaemic heart disease. $B M$ J 1992;303:1295-8.

2 Eysenck HJ, Grossarth-Maticek R, Everitt B. Personality, stress, smoking and genetic predisposition as synergistic risk factors for cancer and coronary
heart disease. Integrative Physiological and Behavioural Science 1991;26: 309-22.

3 Grossarth-Maticek R, Eysenck HJ. Coffee-drinking and personality factors in the genesis of cancer and coronary heart disease. Neuropsychobiology 1990;23:153-9.

4 Grossarth-Maticek R, Eysenck HJ. Personality, stress and motivational factors in drinking as determinants of risk for cancer and coronary heart disease. Psychol Rep 1991;65:1027-43.

5 Grossarth-Maticek R, Eysenck HJ. Coco-cola, cancer and coronaries; personality and stress as mediating factors. Psychol Rep 1991;68:1083-7.

6 Eysenck HJ. Smoking, personality and stress: psychosocial factors in the prevention of cancer and coronary heart disease. New York: Springer Verlag, 1991.

7 Grossarth-Maticek R, Eysenck HJ, Vetter H. Personality type, smoking habit and their interaction as predictors of cancer and coronary heart disease. Personality and Individual Differences 1988;9:479-95.

8 Eysenck HJ. Analysis of mortality data in the 1972 prospective Heidelberg study by Grossarth-Maticek, covering the period 1982-1986. Psychological Inquiry 1991;2:320-1.

Inquiry 1991;2:320-1.
9 Vetter H. Some observations on Grossarth-Maticek's database. Psychological Inquiry 1991;2:286-7.

10 Vetter H. Some genuine predictions of Grossarth-Maticek's established. Psychological Inquiry 1991;2:322-3.

11 Eysenck HJ, Grossarth-Maticek R. Creative novation behaviour therapy as a prophylactic treatment for cancer and coronary heart disease. Part IIEffects of treatment. Behav Res Ther 1991;29:17-31.

12 Eysenck HJ. The prediction of death from cancer by means of personality/ stress questionnaire: too good to be true? Percept Mot Skills 1990;71:216-8.

13 Kissen DM, Eysenck HJ. Personality in male lung cancer patients. fo $_{0}$ Psychosomatic Res 1992;6:123-37.

$14 \mathrm{Schmale} \mathrm{AH}$, Iker H. Hopelessness as a predictor of cervical cancer. Soc Sci Med 1971;5:95-100.

15 Schmitz P. Personality, stress reaction and disease. Personality and Individual Differences 1992;13:683-91.

16 Quander-Blaznik J. Personality as a predictor of lung cancer: a replication. Personality and Individual Differences 1991;12:125-30.

17 Spiegel D, Bloom JR, Kraemer HC, Gottleib E. Effects of psychosocial treatment on sample of patients with metastatic breast cancer. Lancet 1989;ii:888-91.

(Accepted 7 fuly 1992)
What published evidence is there to support the concept of a "golden hour" in the care of patients with major trauma?

The distribution of deaths after major trauma has a trimodal distribution, the first peak of deaths occurring within seconds of injury. The associated injuries are deemed to be unsurvivable and yield the maximum abbreviated injury severity score of 6 . The second peak of deaths occurs within seconds to a few hours after injury. Evidence of avoidable deaths occurring within this period has been obtained from retrospective studies on both sides of the Atlantic.' 'Untreated hypoxia seemed to be a factor in 22 of 170 preventable deaths, and uncorrected haemorrhage was a factor in over half the deaths from causes other than damage to the central nervous system in a British audit of 1000 deaths from trauma. Other studies in the United Kingdom have also identified potentially avoidable factors that have contributed towards deaths during the crucial "golden hour" with estimates varying from $8 \%$ to over $50 \%$. Furthermore, in a study of fatally injured pedestrians the greatest number of deaths, among those who had poten- tially survivable injuries, occurred during the first hour. ${ }^{3}$

Success depends on a team approach with well rehearsed systematic management protocols that can be implemented within the first hour of presentation. The Royal College of Surgeons of England has supported the concept of maximal medical effort, training, and audit through the advanced trauma life support provider and instructor courses. Such courses emphasise the importance of the initial assessment of the victim during the golden hour. Thus with suitably trained staff it should be possible to reduce avoidable deaths during and after the first hour. We are, however, awaiting publication of results from centres in the United Kingdom participating in the major trauma outcome study, which should provide further data to show the importance of the golden hour. ANDREW COPE, consultant in accident and emergency, Peterborough

1 Cales RH, Trunkey DD. Preventable trauma deaths. A review of traum care systems development. FAMA 1985;254:1059-63.

2 Anderson ID, Woodford M, de Dombal FT, Irving M. Retrospective study of 1000 deaths from injury in England and Wales. $B M \mathcal{J}$ 1988;296:1305-8.

3 Fife D. Time from injury to death (survival time) among fatally injured pedestrians. Injury 1987;18:315-8. 